\title{
Evaluation of Fastidious Anaerobe Broth as a blood culture medium
}

\author{
LEELA A GANGULi, LESLEY J TURTON, GLENN S TILLOTSON \\ From the Department of Microbiology, Hope Hospital, Eccles Old Road, Salford M6 8HD
}

SUMMARY Three commercial blood culture media were compared with a freshly prepared cooked meat medium in tests to simulate the recovery of small inocula of anaerobic and aerobic bacteria in routine blood cultures. The cooked meat medium gave the most reliable recovery and supported continued viability, whilst Fastidious Anaerobe Broth (LAB M) was a good alternative. Results with Southern Group thioglycollate and Difco Thiol were less satisfactory as delays in recovery and loss of viability occurred on continued incubation with some of the test strains.

Increasing clinical awareness of the importance of anaerobic infections has resulted in the continuing need for the development of better recovery media, both for purulent material and blood cultures. The superiority of freshly prepared cooked meat medium over various commercially available media for routine blood cultures has previously been reported, but the use of this medium is an extra burden on busy clinical laboratories. The present study was undertaken to compare a new commercial blood culture medium Fastidious Anaerobe Broth (FAB) from LAB M, Ford Lane, Salford, with a freshly prepared cooked meat medium and two other commercial media.

\section{Material and methods}

\section{ORGANISMS}

The following standard strains were used: Bacteroides melaninogenicus ss intermedius, NCTC 9336; Peptostreptococcus anaerobius ATCC 27337; Pseudomonas aeruginosa NCTC 10662; Staphylococcus aureus NCTC 6571 and laboratory clinical isolates of Bacteroides fragilis; Fusobacterium necrophorum, Streptococcus milleri and Streptococcus pneumoniae.

\section{MEDIA}

The following media were bought as prepared blood culture bottles and used according to the manufacturer's instructions. Freshly prepared cooked meat medium was prepared in the laboratory.

(i) Freshly cooked meat medium (CM) was prepared according to Holdeman, Cato and Moore. ${ }^{2}$

Accepted for publication 2 September 1981
Ground, fat-free, beef heart in a ratio of 1 par meat to 4-5 parts of fluid was distributed in $75 \mathrm{~m}$ volumes in $100 \mathrm{ml}$ bottles.

(ii) Fastidious Anaerobe Broth (FAB) from LAB M, Ford Lane, Salford (no product code number available). Bottles contained $75 \mathrm{ml}$ broth.

(iii) Brewer's thioglycollate medium (05.86 CX) from Southern Group Laboratories (SG), Hither Green Hospital, Hither Green Lane, London. Bottles contained $80 \mathrm{ml}$ broth.

(iv) Bacto-Thiol broth (0355-74-2) from Difco Laboratories (Thiol), Central Avenue, West Molesley, Surrey. Bottles contained $100 \mathrm{ml}$ volume with added $\mathrm{CO}_{2}$ under vacuum.

Subcultures were inoculated on to blood agar made with Columbia agar base (LAB M) with $5 \%$ defibrinated horse blood (CBA) medium. Heated blood agar had the same constituents and was heated after the addition of blood. Brain Heart Infusion broth from LAB M (BHI) was presteamed for $10 \mathrm{~min}$ and cooled before being used to prepare the bacterial inoculum.

\section{INOCULUM}

CBA plates were spread with each organism to give confluent growth. These plates were incubated overnight (48 $\mathrm{h}$ for $B$ melaninogenicus). The growth was removed with a swab moistened in BHI and emulsified into $9 \mathrm{ml}$ of BHI. Previous studies showed that this emulsion contained approximately $10^{10}$ colony forming units per $\mathrm{ml}$ (CFU/ml). Serial dilutions were made in BHI and $0.1 \mathrm{ml}$ of the three lowest dilutions-that is, $10^{-6}, 10^{-7}, 10^{-8}$, were used to seed the blood culture bottles. Defibrinated horse blood was added to these bottles to give a final 
concentration of $5 \%$.

Each organism was used at three dilutions and each dilution was also put up in triplicates of the blood culture medium; nine bottles of medium were thus used in tests with each organism. To estimate the variation in inoculum size, $0 \cdot 1 \mathrm{ml}$ of each diluted culture was also placed on to three CBA plates to give a surface count by the spread plate method.

\section{INCUBATION}

All bottles were incubated at $36^{\circ} \mathrm{C}$ on a trolley in a hot room.

\section{SUBCULTURES}

Subculture procedure followed the normal routine in the laboratory, at approximately six hours, then three and seven days. Additional subcultures were done for this project at $24 \mathrm{~h}$ and five days. A sterile $10 \mu 1$ disposable plastic loop (Nunc, Denmark) was used for each subculture except from Thiol broth when a syringe was used according to the manufacturer's specifications.

Subcultures were made by seeding CBA plates which were incubated aerobically and anaerobically (GasPaks in Don Whitley jars). Heated blood agar was also seeded and incubated in $10 \% \mathrm{CO}_{2}$. Growth on these media was recorded subjectively as ranging from a few colonies $(+)$ to heavy confluent growth $(+++)$.

\section{Results}

INOCULUM SIZE

The mean numbers of test bacteria (CFU) inoculated per bottle are shown in Figs 1 and 2. The triplicate plate counts obtained for the minimal inocula were: $B$ fragilis 27, 32, 31; B melaninogenicus 16, 12, 17; $F$ necrophorum 41, 25, 24; Peptostrep anaerobius 5, 4, 9; Staph aureus 6, 9, 9; Ps aeruginosa 2, 3, 10; Str pneumoniae 2, 4, 0; and Str milleri 132, 148, 140.

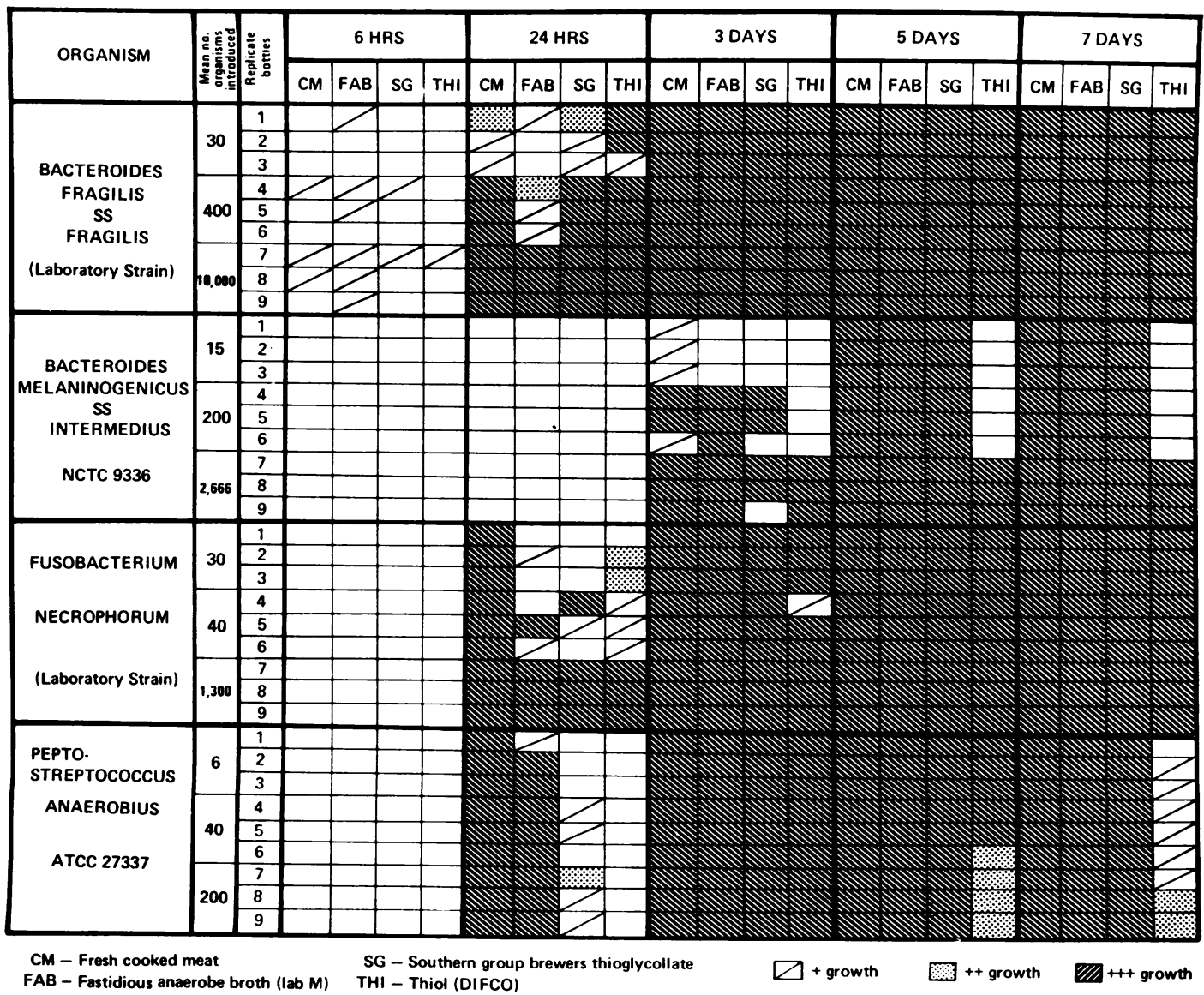

Fig. 1 Recovery of anaerobic test strains from four blood culture media. 


\begin{tabular}{|c|c|c|c|c|c|c|c|c|c|c|c|c|c|c|c|c|c|c|c|c|c|c|}
\hline \multirow{2}{*}{ ORGANISM } & \multirow{2}{*}{ 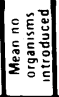 } & \multirow{2}{*}{ 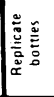 } & \multicolumn{4}{|c|}{$6 \mathrm{HRS}$} & \multicolumn{4}{|c|}{$24 \mathrm{HRS}$} & \multicolumn{4}{|c|}{3 DAYS } & \multicolumn{4}{|c|}{5 DAYS } & \multicolumn{4}{|c|}{7 DAYS } \\
\hline & & & $\mathrm{CM}$ & FAB & SG & THI & $\mathrm{CM}$ & FAB & SG & $T H I$ & $\mathrm{CM}$ & FAB & SG & THI & CM & FAB & SG & THI & CM & FAB & SG & THI \\
\hline \multirow{4}{*}{ STAPHYLOCOCCUS } & & 1 & & & & & & & & & & & & & & & & & & & & \\
\hline & 8 & 2 & & & & & & & & & & & & & & & & & & & & \\
\hline & & 3 & & & & & & & & & & & & & & & & & & & & \\
\hline & & 4 & & & & & & & & & & & & & & & & & & & & \\
\hline AUREUS & 100 & $\frac{5}{6}$ & & & & & & & & & & & & & & & & & & & & \\
\hline \multirow{2}{*}{ NCTC 6571} & & 1 & & & & & & & & & & & & & & & & & & & & \\
\hline & 2,000 & 8 & & & & & & & & & & & & & & & & & & & & \\
\hline \multirow{4}{*}{ PSEUDOMONAS } & & 1 & & & & & & & & & & & & & & & & & & & & \\
\hline & 5 & 2 & & & & & & & & & & & & & & & & & & & & \\
\hline & & $\frac{3}{4}$ & & & & & & & & & & & & & & & & & & & & \\
\hline & & 4 & & & & & & & & & & & & & & & & & & & & \\
\hline \multirow{2}{*}{ AERUGINOSA } & 200 & 5 & & & & & & & & & & & & & & & & & & & & \\
\hline & & 7 & & & & & & & & & & & & & & & & & & & & \\
\hline \multirow[t]{2}{*}{ NCTC 10662} & 1.000 & 8 & & & & & & & & & & & & & & & & & & & & \\
\hline & & 9 & & & & & & & & & & & & & & & & & & & & \\
\hline \multirow{4}{*}{ STREPTOCOCCUS } & & 1 & & & & & & & & & & & & & & & & & & & & \\
\hline & 2 & 2 & & & & & & & & & & & & & & & & & & & & \\
\hline & & 3 & & & & & & & & & & & & & & & & & & & & \\
\hline & & 4 & & & & & & & & & & & & & & & & & & & & \\
\hline \multirow[t]{2}{*}{ PNEUMONIAE } & 30 & 5 & & & & & & & & & & & & & & & & & & & & \\
\hline & & 6 & & & & & & & & & & & & & & & & & & & & \\
\hline \multirow{2}{*}{ (Laboratory Strain) } & & 7 & & & & & & & & & & & & & & & & & & & & \\
\hline & 500 & $\frac{8}{9}$ & & & & & & & & & & & & & & & & & & & & \\
\hline \multirow{9}{*}{ STREPTOCOCCUS } & & 1 & & & & & & & & & & & & & & & & & & & & \\
\hline & 140 & 2 & & & & & & & & & & & & & & & & & & & & \\
\hline & & 3 & & & & & & & & & & & & & & & & & & & & \\
\hline & & 4 & & & & & & & & & & & & & & & & & & & & \\
\hline & 1,500 & 5 & & & & & & & & & & & & & & & & & & & & \\
\hline & & 6 & & & & & & & & & & & & & & & & & & & & \\
\hline & 8 & 7 & & & & & & & & & & & & & & & & & & & & \\
\hline & మ్ల & 8 & & & & & & & & & & & & & & & & & & & & \\
\hline & $\underline{m}$ & 9 & & & & & & & & & & & & & & & & & & & & \\
\hline
\end{tabular}

Fig. 2 Recovery of aerobic test strains from four blood culture media.

The blood culture results are presented for anaerobic organisms (Fig. 1) and aerobic organisms (Fig. 2).

An interesting point was that $B$ melaninogenicus was detected on subculture before either visible turbidity or haemolysis was observed in any medium.

\section{Discussion}

Overall, the cooked meat medium $(\mathrm{CM})$ was the best medium both for initial recovery and sustained viability of all test organisms. FAB was seen to be a reliable medium only slightly inferior to $C M$ under these test conditions. FAB was superior to $\mathrm{CM}$ in that $B$ fragilis and Str pneumoniae were recovered after incubation for only six hours. Results with Brewer's thioglycollate (SG) and Bacto-Thiol were less satisfactory under these test conditions.

Our CM results agree with those reported by Collee et $a l^{1}$ and $\mathrm{Barr}^{3}$ using this medium. As can be seen from Figs 1 and 2, all organisms can be readily recovered and remained viable throughout the tests. Disadvantages associated with CM are that its preparation is both time and space-consuming, adding an extra burden to a routine laboratory. The lack of clarity of the sterile medium also presents problems in judging visible evidence of growth.

Brewer's thioglycollate medium (SG) was recommended in ACP Broadsheet 81 for anaerobic blood culture. ${ }^{4}$ A survey in 1972 of media used in Britain showed SG thioglycollate to be the best available.5 In the present study the recovery of aerobic and anaerobic streptococci in thioglycollate broth (SG) was delayed and the viability of these strains was markedly decreased. Szawatkowski ${ }^{6}$ also reported the delayed recovery and loss of viability of $B$ fragilis strains in Brewer's thioglycollate, although the medium manufacturer was not mentioned.

Results with Thiol broth (Difco) were poor; in particular rapid recovery of anaerobes and Str 
pneumoniae were not possible and also $B$ melaninogenicus was only recovered from heavy inocula. The ability of this medium to recover Ps aeruginosa is unreliable, but Difco make no claims as to the effectiveness of Thiol broth to support the growth of aerobes. Szawatkowski ${ }^{6}$ reported Difco Thiol to be superior to thioglycollate broth and commercial cooked meat broth for the rapid isolation of various Bacteroides spp and Fusobacterium spp. Shanson and Barnicoat ${ }^{7}$ also found this medium to be a satisfactory alternative to SG thioglycollate for the isolation of anaerobes. Our results agree with those of Collee $e t a^{1}$ and Barr $^{3}$ who found it less reliable for the isolation of anaerobic bacteria.

Initial studies were done to determine the recovery of small inocula of $B$ melaninogenicus from various anaerobic media (Turton and Ganguli, unpublished observations). The media tested included various thioglycollates-for example, United States Pharmacopeia (USP), Brewer's, and a fluid thioglycollate medium-all supplied by LAB M - and Brewer's thioglycollate from Baltimore Biological Laboratories, Division of Becton, Dickinson and Co, Cockeysville, Maryland 21030, USA and a cooked meat medium with $\mathrm{BHI}$ overlay $(\mathrm{CM} / \mathrm{BHI})$ from LAB $M$. The best medium for the recovery and viability of the test organism was CM/BHI. USP thioglycollate gave rapid recovery of small inocula of $B$ melaninogenicus, but loss of viability occurred within five days of inoculation. Thus, to prevent loss of viability, USP thioglycollate was modified in collaboration with LAB M to develop Fastidious Anaerobe Broth (Porter and Hyde, unpublished observations). The relative costs for 100 bottles of the media tested are as follows (January 1981):

Thiol (Difco) 0355-74-2, £52.80 + VAT + carriage, bottles disposable; Southern Group Brewer's thioglycollate 0586CX, $£ 33.00$ + carriage, bottles returnable; FAB (LAB M), no code number available, $£ 31.00$ + VAT + carriage, bottles disposable. The cost of freshly prepared cooked meat was $£ 22.29$ which includes $£ 6.46$ for reusable bottles; however, this price makes no allowance for technician time or laboratory overheads.

It is widely recommended that at least two media, one for anaerobes and another for aerobes, are used in order to recover the majority of organisms from infected blood. It is of additional benefit to use an anaerobic isolation medium which can also support the growth of aerobes. It is frustrating that cooked meat medium is still superior, but FAB appears to be a good medium for the recovery of both anaerobic and aerobic bacteria from blood cultures.

We thank William Hyde and Wendy O'Hare of the Microbiology Department for their technical assistance. GS Tillotson wishes to thank the Wellcome Trust for financial support.

\section{References}

${ }^{1}$ Collee JG, Duerden BI, Brown R. Recovery of anaerobic bacteria from small inocula: a model for blood culture studies. J Clin Pathol 1977;30:609-14.

${ }^{2}$ Holdeman LV, Cato EP, Moore WEC. Anaerobe laboratory manual. 4th ed. Blacksburg, Virginia: Virginia Polytechnic Institute and State University, 1977.

${ }^{3}$ Barr JG. A cooked meat blood culture medium; shelf life and clinical evaluation compared with other systems. $J$ Infect $1980 ; 2: 247-58$.

${ }^{4}$ Stokes EJ. Blood culture technique. ACP Broadsheet 81 , 1974.

${ }^{5}$ Shanson DC. An experimental assessment of different anaerobic blood culture methods. J Clin Pathol 1974; 27:273-9.

${ }^{6}$ Szawatkowski MV. A comparison of three readily available types of anaerobic blood culture media. Med Lab Sci 1976;33:5-12.

${ }^{7}$ Shanson DC, Barnicoat M. An experimental comparison of Thiol broth with Brewer's thioglycollate for anaerobic blood cultures. J Clin Pathol 1975;28:407-9.

Requests for reprints to: Dr LA Ganguli, Department of Microbiology, Hope Hospital, Eccles Old Road, Salford M6 8HD, England. 